\title{
Exchange interaction-driven dynamic nuclear polarization in Mn-doped InGaAs/GaAs quantum dots
}

\author{
O. Krebs, ${ }^{1}$ E. Baudin, ${ }^{2}$ and A. Lemaître ${ }^{1}$ \\ ${ }^{1}$ Centre de Nanosciences et de Nanotechnologies, CNRS, Université Paris-Sud, Université Paris-Saclay, \\ C2N-Marcoussis, 91460 Marcoussis, France \\ ${ }^{2}$ Laboratoire Pierre Aigrain, Ecole Normale Supérieure, PSL Research University, CNRS, Université Pierre et Marie Curie, Sorbonne \\ Universités, Université Paris Diderot, Sorbonne Paris-Cité, 24 rue Lhomond, 75231 Paris Cedex 05, France
}

(Received 2 August 2016; published 10 November 2016)

\begin{abstract}
We investigated optical spin orientation and dynamic nuclear polarization (DNP) in individual self-assembled InGaAs/GaAs quantum dots (QDs) doped by a single Mn atom, a magnetic impurity providing a neutral acceptor $A^{0}$ with an effective spin $J=1$. We find that the spin of an electron photocreated in such a quantum dot can be efficiently oriented by a quasiresonant circularly polarized excitation. For the electron spin levels which are made quasidegenerate by a magnetic field compensating the exchange interaction $\Delta_{e}$ with $A^{0}$, there is however a full depolarization due the anisotropic part of the exchange. Still, in most studied QDs, the spin polarized photoelectrons give rise to a pronounced DNP which grows with a longitudinal magnetic field until a critical field where it abruptly vanishes. For some QDs, several replica of such DNP sequence are observed at different magnetic fields. This striking behavior is qualitatively discussed as a consequence of different exchange interactions experienced by the electron, driving the DNP rate via the energy cost of electron-nucleus spin flip-flops.
\end{abstract}

DOI: 10.1103/PhysRevB.94.195412

\section{INTRODUCTION}

Semiconductor quantum dots (QDs) doped by a single or few magnetic impurities have been studied in the last decade in order to investigate the exchange interaction between spin carriers in the quantum regime [1-9], and the potential of such a system as a solid-state quantum bit [10-19]. In such QDs the dominant two-spin interaction is the exchange interaction between the magnetic dopant and the QD-confined hole (up to a few $\mathrm{meV})$. Then comes the electron-hole exchange interaction $(\sim 0.5 \mathrm{meV})$ and the exchange interaction between the magnetic dopant and the QD-confined electron $(\sim 0.1 \mathrm{meV}$ or less). In this context, the hyperfine interaction of the confined carriers with the $\sim 10^{4}-10^{5}$ nuclear spins of the QD matrix, with typical fluctuations in the $\mu \mathrm{eV}$ range, turns out to be a small perturbation. However, it has been proven to play an essential role for the spin dynamics of a single electron in undoped QDs [20], in particular through the ability, under various experimental conditions, to strongly polarize the nuclear spins [21-26]. This raises the question whether any such manifestation of the hyperfine interaction between a single electron and the nuclear spin bath can be observed in magnetically doped QDs.

In this paper we address this issue by focusing more specifically on the electron- $A^{0}$ system in single InGaAs/GaAs QDs where $A^{0}$ represents the neutral magnetic acceptor, with an effective spin $J=1$, provided by a substitutional Mn impurity in the InGaAs matrix together with its bound hole $[5,27,28]$. Our experiments reveal that the exchange interaction with the magnetic impurity $A^{0}$ drastically perturbs the mechanism of dynamic nuclear polarization (DNP) by a spin-polarized electron. It leads either to a partial inhibition or, more surprisingly, to a succession of DNP increases developing when a longitudinal magnetic field is swept. These observations can be qualitatively interpreted as a function of the strength and anisotropy of the electron- $A^{0}$ exchange interaction.

\section{SAMPLES AND EXPERIMENTAL METHODS}

In the following we report a set of observations carried out on four distinct Mn-doped InGaAs/GaAs QDs (labeled QD $n$, with $n=1$ to 4 ) originating from two different samples: QD1 is in a sample consisting of a single layer of InGaAs/GaAs QDs with a low p-type residual doping [6], while QD2, QD3, and QD4 are from a diode sample where the Mn-doped QD layer is coupled to an electron reservoir enabling the charge control by an applied electrical bias [5,13]. Let us recall that due to the temperature required for the $\mathrm{QD}$ growth the effective Mn doping remains quite low with typically less than $1 \%$ of the QDs showing an actual coupling with an $A^{0}$ impurity. Thus, the QDs of the present study were first sought by scanning some sample areas with a microphotoluminescence ( $\mu$-PL) setup. They were confirmed as Mn-doped QDs thanks to their specific spectral signature in a magnetic field [5]. Our $\mu$-PL setup relies on a 2-mm focal length aspheric lens (0.5 NA) actuated by piezostages and mounted in a split-coil magneto-optical cryostat. The optical excitation is provided either by a HeNe laser or a continuous wave (cw) tunable Ti-sapphire laser. The collected PL is analyzed with a set of linear and quarter-wave plates to resolve its circular $\sigma^{+}$ or $\sigma^{-}$polarization. It is then dispersed by a $0.6-\mathrm{m}$ focal length double spectrometer equipped with a nitrogen-cooled CCD array camera providing a multichannel detection with $\approx 10 \mathrm{meV}$ spectral range and typical $15 \mathrm{~s}$ integration times. All measurements were performed at low temperature $(\leqslant 5 \mathrm{~K})$ and the magnetic field was applied parallel to the optical and QD growth axis $z$.

The QDs have been studied in a regime where they are positively charged by an additional hole, as evidenced by their magneto-optical signature [6]. In the diode sample this relies on an optical charging which takes place when the electron of a photocreated neutral exciton tunnels out of the dot, due to a high internal electric field [21]. Under optical excitation, 
positive trions $X^{+}$(two holes, one electron) are thus created. In such a complex, both holes are paired in a singlet, so that their spin-related interactions with other particles vanish. The $X^{+}$ spin thus corresponds to the spin of the photocreated electron with eigenstates $\left|S_{z, e}= \pm 1 / 2\right\rangle$ (also denoted $\uparrow$ or $\downarrow$ ), which interacts with the $A^{0}$ spin and the QD nuclear spins during the trion's lifetime. Thanks to the optical selection rules of trions in QDs (inherited from the heavy hole spin-orbit coupling), it can be analyzed via the PL circular polarization which reads $\mathcal{P}_{\mathrm{c}}=2\left\langle S_{z, e}\right\rangle=\left(I_{\sigma-}-I_{\sigma+}\right) /\left(I_{\sigma-}+I_{\sigma+}\right)$, where $I_{\sigma \pm}$ is the PL intensity detected in $\sigma \pm$ polarization.

\section{SPIN POLARIZATION IN MAGNETIC FIELD}

In undoped QDs, exciting an $X^{+}$trion with circularly polarized light offers a direct means to investigate the hyperfine interaction with the nuclei and possibly to induce efficient dynamic nuclear polarization [20]. For example, changes in PL circular polarization can reveal electron spin relaxation induced by the transverse fluctuations of the so-called Overhauser field (i.e., the nuclear spin polarization), whereas the spectral splitting of the PL circular components in zero field, or its shift with respect to normal Zeeman splitting in a magnetic field $B_{z}$, reflects the average value of this Overhauser field along $z$. To apply the same approach to the case of Mn-doped QDs, we first need to carefully analyze the electron- $A^{0}$ system, namely its level structure, the spin eigenstates, and the intrinsic spin polarization which may develop due to magnetic field and exchange interactions with $A^{0}$ in the absence of optically induced spin orientation or pumping.

Figure 1 presents such a preliminary investigation of QD1 achieved under nonpolarized and nonresonant excitation (633 nm HeNe laser line, $10 \mu \mathrm{W}$ incident power). In Fig. 1(a) the characteristic polarization-resolved magneto-PL spectra of such Mn-doped InGaAs QD is shown: it consists of two main lines, corresponding to the trion transitions with constant $A^{0}$ spin states $| \pm 1\rangle$, which anticross with two weaker lines corresponding to "forbidden" transitions where the $A^{0}$ spin is flipped $(| \pm 1\rangle \rightarrow|\mp 1\rangle)$. Altogether they form a remarkable X-pattern. The theoretical levels corresponding to these transitions are plotted in Figs. 1(b) and 1(c). Detailed discussions about such images and the model Hamiltonians enabling us to calculate the levels can be found in Refs. $[6,18]$. Here we mostly focus on the $A^{0}$ and trion spin polarization which results from the thermal relaxation taking place both in the $A^{0}-X^{+}$configuration (transition initial state) and in the $A^{0}$ hole configuration (final state), as illustrated by wavy arrows in Figs. 1(b) and 1(c). The experimental trion polarization (i.e., electron spin polarization) can be easily deduced from Fig. 1(a) by integrating separately the $\sigma^{+}$and $\sigma^{-}$PL intensity over a typical $3 \mathrm{meV}$ spectral range. To estimate the $A^{0}$ spin polarization, namely the ratio $\left(p_{+1}-p_{-1}\right) /\left(p_{+1}+p_{-1}\right)$, where $p_{ \pm 1}$ represents the $A^{0}$ population in the state $| \pm 1\rangle$, we extract with an appropriate line fit the total intensity of the PL lines associated either with a $|+1\rangle$ or $a|-1\rangle$ state, which are assumed to be proportional to the corresponding populations. The results are shown in Fig. 1(d).

As previously observed [1,5], the $A^{0}$ spin acquires a strong polarization (negative in positive fields) when the magnetic field $B_{z}$ increases. This behavior can be fairly
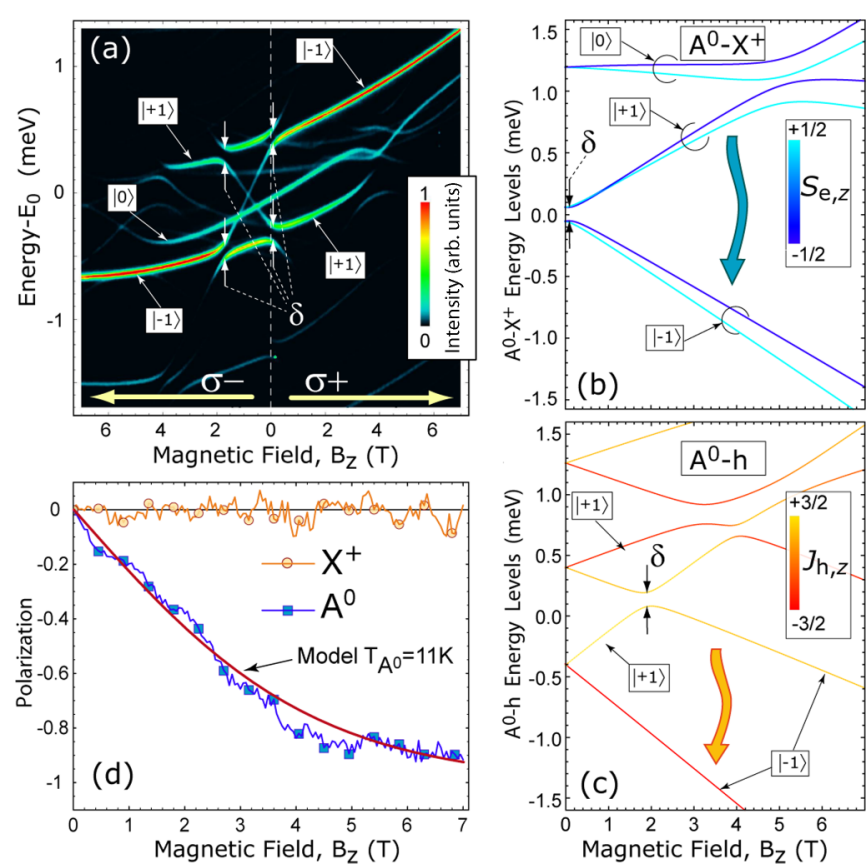

FIG. 1. (a) QD1 PL spectra measured in circular polarization ( $\sigma^{+}$or $\sigma^{-}$as indicated) as a function of the magnetic field $B_{z}$ under nonresonant unpolarized excitation. $E_{0}=1.341 \mathrm{eV}$ and $T=5 \mathrm{~K}$. (b) and (c) Calculated energy levels of the initial state $\left(A^{0}-X^{+}\right)$and final state $\left(A^{0}-\mathrm{h}\right)$ providing a good simulation of (a) (not shown). The wavy arrows illustrate the expected relaxation leading to a large spin polarization. (d) Polarization of $A^{0}$ spin and $X^{+}$PL against magnetic field. The solid line is calculated according to Brillouin's model of thermalization for a $1 / 2$ spin.

well reproduced by the Brillouin function of a $1 / 2$ spin, $B_{1 / 2}\left(B_{z}\right)=\tanh \left(g_{A^{0}} \mu_{\mathrm{B}} B_{z} / k_{\mathrm{B}} T_{A^{0}}\right)$, where $g_{A^{0}}=3.6$ is the $A^{0}$ $g$ factor determined from the X-pattern in Fig. 1(a), $\mu_{\mathrm{B}}$ is the Bohr magneton, $k_{\mathrm{B}}$ is the Boltzmann constant, and $T_{A^{0}}=11 \mathrm{~K}$ is the $A^{0}$ temperature. This temperature, considered here as a fitting parameter, is slightly higher than the lattice temperature $(5 \mathrm{~K})$, likely due to the optical excitation and recombination with $A^{0}$ spin-flip. It still indicates an efficient spin relaxation to the two lowest levels of $A^{0}-X^{+}$leading to an $A^{0}$ spin polarization of $-90 \%$ at $B_{z}=7 \mathrm{~T}$.

In contrast, the $X^{+}$polarization remains essentially equal to zero, although the electron thermal polarization in $X^{+}$should amount to $25 \%$ at $7 \mathrm{~T}$ and $5 \mathrm{~K}$, according to the electron $g$-factor $g_{e}=-0.48$ in QD1 [deduced from Fig. 1(a)]. This indicates that the electron spin relaxation is likely inefficient during the $X^{+}$lifetime. This absence of $X^{+}$polarization also implies that the polarization of the resident hole, which is presumably quite high because of the large $A^{0}$-hole exchange interaction [see Fig. 1(c)], is not transferred to the electron when an $X^{+}$is created nonresonantly.

From these preliminary observations, it can thus be assumed that the $X^{+}$polarization will reflect with fidelity the spin state of the electron captured or photocreated in the QD and its intrinsic subsequent evolution, in particular under the influence of the hyperfine interaction or the electron- $A^{0}$ exchange. In that respect, the field $\left|B_{\delta}\right| \approx 79 \mathrm{mT}$ of the $\delta$ anticrossing in Fig. 1(b) provides a direct estimate of the exchange strength $\Delta_{e-A^{0}}=$ 
$2 g_{A^{0}} \mu_{\mathrm{B}}\left|B_{\delta}\right| \approx 33 \mu \mathrm{eV}$. In principle, this should protect the electron spin from the nuclear spin bath fluctuations. However, near zero field, the actual electron spin splitting is significantly reduced because of the strong mixing of the $|+1\rangle$ and $|-1\rangle A^{0}$ states due to the $\delta / 2 \approx 72 \mu \mathrm{eV}$ coupling. It is thus difficult to predict if the small hyperfine interaction with the nuclei will induce some spin relaxation and/or initiate a nuclear spin polarization which then could be amplified by a magnetic field as observed for undoped QDs [20].

\section{CORRELATED $A^{0}$-TRION SPIN POLARIZATION}

In order to observe a high degree of $X^{+}$circular polarization, InGaAs QDs have to be excited more resonantly, at least below the $\sim 1.42 \mathrm{eV}$ wetting layer band gap. We first performed the PL excitation spectroscopy of QD1 with a tunable cw Ti:sapphire in circular polarization, see Fig. 2(a). Note that the PL intensity has been normalized by the incident laser power which was varying from $\sim 1$ to $\sim 4 \mathrm{~mW}$ when
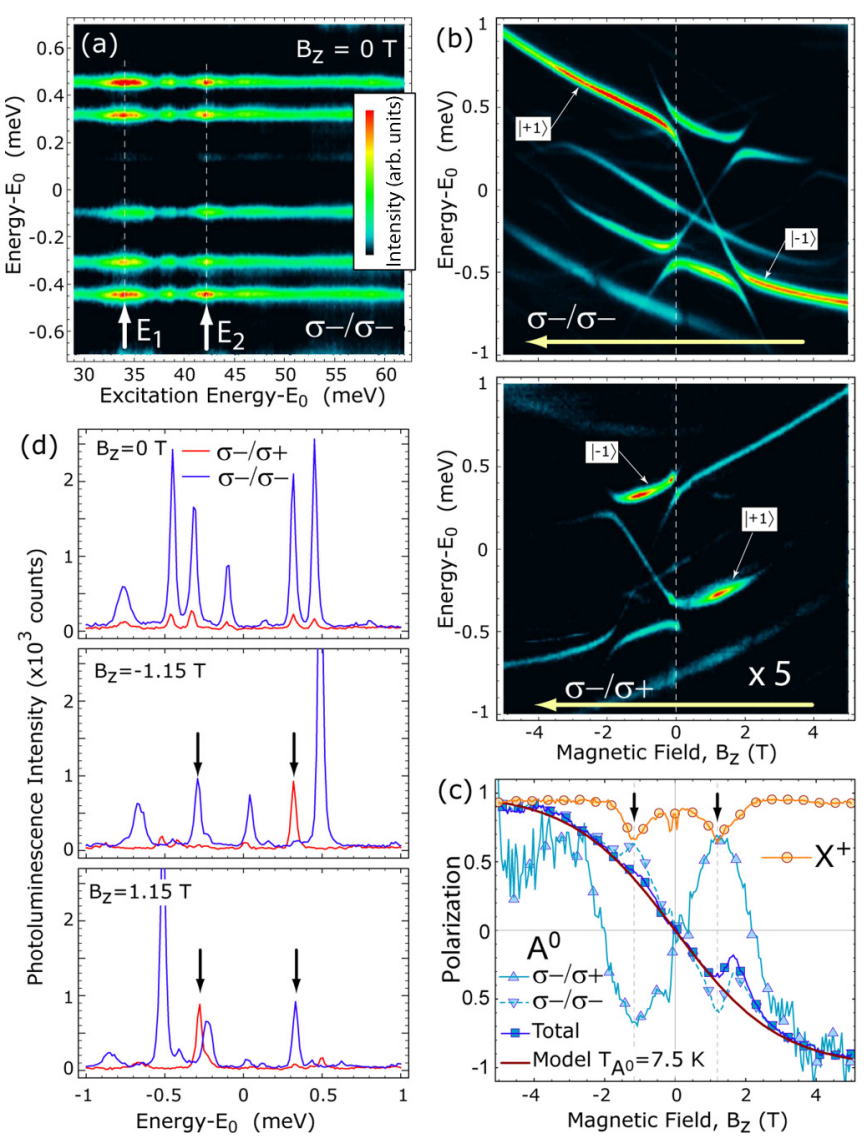

FIG. 2. (a) QD1 PL spectra measured in copolarized configuration $(\sigma-/ \sigma-)$ as a function of the excitation energy. (b) PL spectra under quasiresonant $\sigma-$ excitation [resonance $E_{1}$ in (a)] as a function of a decreasing magnetic field $B_{z}$ and measured in $\sigma-$ (top) or $\sigma+$ (bottom, $\times 5$-amplified) polarization. (c) Polarization of $A^{0}$ spin for $\sigma+(\triangle), \sigma-(\nabla)$ or both measurements $(\square)$ and $X^{+}$PL $(\bigcirc)$ against magnetic field deduced from (b). $A^{0}$ polarization conditioned to the co- or cross-polarized measurements shows strong correlations with the trion PL polarization. (d) PL spectra at three specific magnetic fields. The co- and cross-polarized lines marked by arrows exhibit the same intensity at $B_{z}= \pm 1.15 \mathrm{~T}$. increasing the excitation energy. Two resonances $E_{1}$ and $E_{2}$ were found, respectively, 34 and $42 \mathrm{meV}$ above the central PL energy $E_{0}=1.341 \mathrm{eV}$ of QD1, both providing a noticeable polarization above $80 \%$ in zero field. This first result indicates that, like in undoped QDs, the electron spin of $X^{+}$is well protected from the relaxation caused by the fluctuations of the hyperfine interactions, whatever is the reason (strong electron- $A^{0}$ exchange or DNP-induced Overhauser field). Note that the above resonances are relatively large $(\geqslant 1 \mathrm{meV})$ and do not select any specific $A^{0}$ spin state, at least in zero field.

We then investigated how the $\mathrm{X}^{+}$optical orientation evolves in a magnetic field. Figure 2(b) shows the magneto-PL images obtained under $\sigma^{-}$excitation at $E_{1}$ when varying the magnetic field from +5 to $-5 \mathrm{~T}$ for both copolarized $\left(\sigma^{-} / \sigma^{-}\right)$ and cross-polarized $\left(\sigma^{-} / \sigma^{+}\right)$detections. The latter, which is plotted with a $\times 5$-amplified color scale, evidences noticeable enhancements around the two magnetic fields of $\pm 1.15 \mathrm{~T}$. This likely indicates local reductions of $X^{+}$polarization around these fields, which is confirmed by extracting from the integrated spectra the whole $X^{+}$polarization. Two pronounced dips of about $20 \%$ amplitude appear around these fields as indicated by arrows in Fig. 2(c).

What is quite remarkable is that the enhancement of the $\sigma^{-} / \sigma^{+}$PL signal is clearly correlated to a specific $|+1\rangle$ or $|-1\rangle A^{0}$ state. This behavior looks like a strong polarization of $A^{0}$ opposite in sign to that resulting from the usual thermalization. To analyze these observations more quantitatively, we extracted in Fig. 2(c) the $A^{0}$ polarization separately for the cross- or copolarized configurations, as well as the total $A^{0}$ polarization deduced from both sets of measurements. They are plotted together with a Brillouin function at an effective temperature $T_{A^{0}}=7.5 \mathrm{~K}$. Note that due to the overall strong PL polarization, the total $A^{0}$ polarization is very similar to the $A^{0}$ polarization extracted from the copolarized configuration. The strong inversion of $A^{0}$ polarization in cross-polarized configuration, up to $\sim \pm 70 \%$ at $\pm 1.15 \mathrm{~T}$, appears to be essentially compensated by a small increase of the normal polarization (in absolute value) in the copolarized one: the total $A^{0}$ polarization indeed no longer shows any significant anomaly with respect to the Brillouin function at these fields. There is still a polarization reduction around $+1.7 \mathrm{~T}$, but this effect most likely results from the $\delta$ anticrossing which induces the total mixing of the $|+1\rangle$ and $|-1\rangle A^{0}$ states occurring for the $\sigma^{-}$polarized transition toward the hole spin state $J_{z}=+3 / 2$, see Fig. 1(c). Incidentally, this absence of anomaly on the total $A^{0}$ polarization associated with the depolarization of $X^{+}$ allows us to exclude an electron-induced spin orientation of $A^{0}$, in contrast to the Mn spin in magnetic CdTe/ZnTe QDs [10], as the cause of this correlation.

To elucidate the origin of this intriguing correlation, the calculated $A^{0}-X^{+}$levels in Fig. 1(b) turn out very helpful. It is noteworthy that the two electron spin levels (with $S_{e, z}= \pm 1 / 2$ ) associated with the $|+1\rangle A^{0}$ state remain very close up to $\sim 1.5 \mathrm{~T}$ with most probably a crossing at about $1 \mathrm{~T}$, corresponding to the exchange field $B_{\Delta}=\Delta_{e-A^{0}} / g_{e} \mu_{\mathrm{B}}$. The symmetrical situation (not shown) holds at about $-1 \mathrm{~T}$ for the levels associated with the $|-1\rangle A^{0}$ state. Since the spin relaxation by energy conserving mechanisms (like hyperfine interaction) is favored when the electron spin splitting vanishes, such crossings certainly point to the origin of our 
observations. Experimentally, the electron spin splitting cannot be directly observed because the two allowed transitions from these $S_{e, z}= \pm 1 / 2$ levels with, respectively, a $J_{z}=\mp 3 / 2$ hole are split by the strong $A^{0}$-hole exchange in the final state. We can still compare the intensities of the corresponding lines to assess more quantitatively the amount of electron spin relaxation between the two levels. Figure 2(d) shows three cross sections of the magneto-PL images on a common vertical scale. Whereas in zero field the $X^{+}$polarization is strong for all the QD1 transitions, at $\pm 1.15 \mathrm{~T}$ the two $X^{+}$lines corresponding to the $| \pm 1\rangle A^{0}$ state (marked by a dark arrow) exhibit essentially the same intensity in cross- and copolarized measurements. Obviously the electron spin relaxation taking place at these crossing points is very high (if not total).

This result actually discards the hyperfine interaction with the nuclei as the dominant mechanism for the spin relaxation, because the depolarization by nuclei, even for degenerate electron spin states, would be limited to $\sim 50 \%$ of its initial value over the $\sim 1 \mathrm{~ns}$ trion lifetime [20]. By inspecting closer the two calculated levels associated with a same $| \pm 1\rangle A^{0}$ state, we found out that actually they anticross by an energy $\delta_{e} \approx 9 \mu \mathrm{eV}$ for QD1. In this region, the $X^{+}$eigenstates are totally mixed spin states, and since $\delta_{e}$ is significantly larger than the $X^{+}$natural width, the average spin polarization vanishes. The origin of this anticrossing is obviously related to the electron- $A^{0}$ exchange and to the lack of perfect rotational symmetry of the system, in a way similar to the previously observed dark-bright mixing of the $A^{0}$-hole spin levels $[5,6,29]$. This will be discussed in more detail below with the measurements on QD2 for which the $\delta_{e}$ anticrossing could be experimentally resolved.

\section{FINE ANTICROSSING OF $\boldsymbol{A}^{\mathbf{0}}$-TRION LEVELS}

QD2 in Fig. 3(a) exhibits a particularly strong exchange interaction with the $A^{0}$ impurity characterized by a $\simeq 1.4 \mathrm{meV}$ splitting between the $|+1\rangle$ and $|-1\rangle$ lines. From the field $\left|B_{\delta}\right|=215 \mathrm{mT}$ of the $\delta$ anticrossing and the $A^{0} g$-factor $g_{A^{0}}=2.8$ deduced from the X-pattern, we can extract the contribution due to the electron- $A^{0}$ exchange energy to $\Delta_{e-A^{0}}=2 g_{A^{0}} \mu_{\mathrm{B}}\left|B_{\delta}\right| \approx 70 \mu \mathrm{eV}$. Although it is about twice larger than for QD1, there is no strong enhancement of the cross-polarized PL lines associated with the $|+1\rangle$ or $|-1\rangle A^{0}$ states near the field where the levels are expected to anticross. Instead, we observe a clear splitting of the $| \pm 1\rangle$ lines, both in co- and cross-polarized configurations, over the magnetic field range from basically $0 \mathrm{~T}$ up to about $\pm 3 \mathrm{~T}$. The PL spectra in Fig. 3(b) illustrate this feature at the fields of $\pm 1.6 \mathrm{~T}$ where the split lines of each $\delta_{e}$ doublets have roughly the same intensity and the $\delta_{e}$ splitting reaches a minimum value of $47 \mu \mathrm{eV}$.

These observations are in good agreement with the prediction of our simplest spin model [18] based on an effective $J=1$ for $A^{0}$, as shown by the calculated levels in Fig. 3(c). An important parameter of this model, which notably explains the drastic difference between QD1 and QD2, is the inclination by an angle $\theta_{s}$ of the dominant strain field experienced by the $A^{0}$ impurity with respect to the QD growth axis. For QD2 the angle $\theta_{s}$ deduced from the simulation of the magneto-PL images is estimated to be $33^{\circ}$ while it amounts to only $13^{\circ}$ for QD1. It allows for the coupling of the $|+1, \uparrow\rangle(|-1, \downarrow\rangle)$
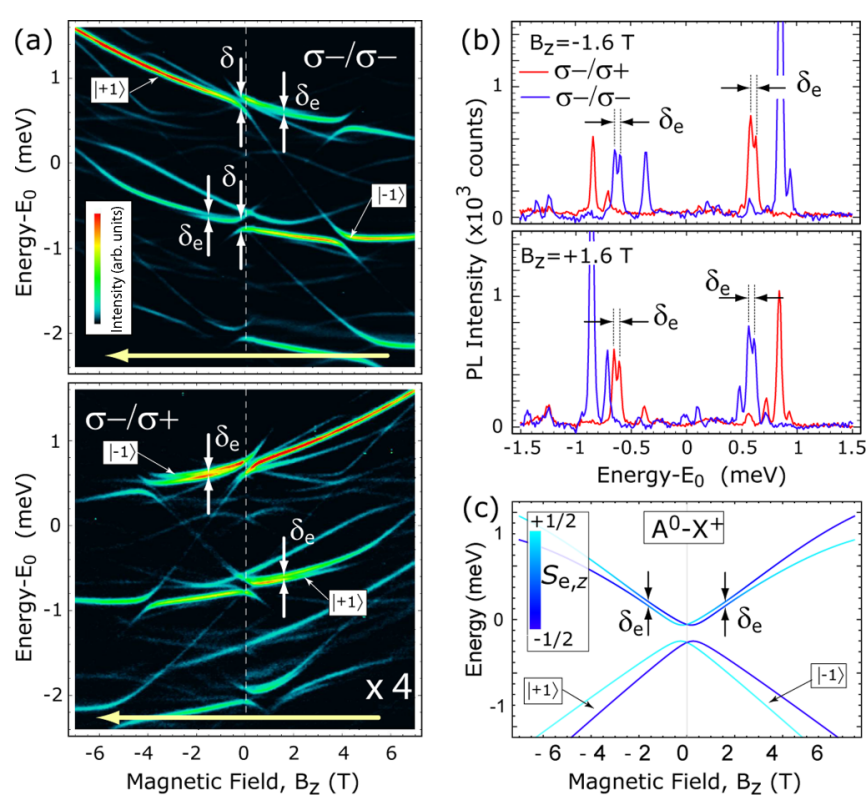

FIG. 3. (a) QD2 PL spectra under quasiresonant $\sigma$ - excitation at $E_{0}+60 \mathrm{meV}$ as a function of a decreasing magnetic field $B_{z}$ and measured in $\sigma-$ (top) or $\sigma+$ (bottom, $\times 4$-amplified) polarization. ( $E_{0}=1.281 \mathrm{eV}$.) White arrows point out spectrally resolved anticrossings. (b) PL spectra at $B_{z}= \pm 1.6 \mathrm{~T}$ showing the splitting $\delta_{e} \approx 47 \mu \mathrm{eV}$ of the anticrossing transitions responsible for the reduction of PL polarization. (c) Calculated energy levels of the corresponding $e-A^{0}$ states.

$A^{0}$-electron state to $|0, \uparrow\rangle(|0, \downarrow\rangle)$ which is also coupled by the (Heisenberg-like) electron- $A^{0}$ exchange to $|+1, \downarrow\rangle(|-1, \uparrow\rangle)$. Both terms induce a coupling of the $\uparrow$ and $\downarrow$ electron spin states associated with the same $|+1\rangle$ or $|-1\rangle$ state, which can be seen as an effective in-plane magnetic field $\delta_{e} /\left(g_{e} \mu_{\mathrm{B}}\right)$ and gives rise to the observed anticrossing. To the first order in perturbation theory, we indeed find that the $\delta_{e}$ splitting is given by $2 \Delta_{e-A^{0}} \sin 2 \theta_{s} /\left(3+\cos 2 \theta_{s}\right)$ which provides good estimates of the $\delta_{e}$ splitting for QD1 (7.4 $\left.\mu \mathrm{eV}\right)$ and QD2 (40 $\left.\mu \mathrm{eV}\right)$ from the fitted angle $\theta_{s}$.

From the above analysis, it seems obvious that the electron$A^{0}$ exchange in Mn-doped QDs must strongly inhibit the DNP mechanism with spin-oriented electrons: to experience a spin flip-flop with a nucleus with a reasonable probability the two electron spin states have to be close enough in energy [20], but in this case the electron spin, optically oriented along $z$, tends to vanish due to the $\delta_{e}$-induced coupling, see Fig. 3(c). In zero field, this conclusion can be slightly revised because of the strong mixing of the $|+1\rangle$ and $|-1\rangle$ states. The electron- $A^{0}$ eigenstates form two Kramers doublets split by $\left(\delta^{2}+\Delta_{e-A^{0}}^{2}\right)^{1 / 2}$ and reading $\mid+, \uparrow$ or $\left.\downarrow\right\rangle$ and $\mid-, \uparrow$ or $\downarrow\rangle$ where $| \pm\rangle \simeq(|+1\rangle \pm|-1\rangle) / \sqrt{2}$. Within each of these doublets the electron can experience spin flip-flops with a nucleus without any energy cost, leading possibly to DNP. In the above experiments, by comparing the co- and cross-polarized spectra in zero field, we could indeed observe for both QD1 and QD2 a finite Overhauser shift amounting to about $15 \mu \mathrm{eV}$, very similar to that observed in undoped QDs. Furthermore, after a careful analysis of the data as detailed below, it turns out that this shift actually survives up to 
$\approx 0.5 \mathrm{~T}$ while increasing up to $\approx 25 \mu \mathrm{eV}$, and then abruptly vanishes, most likely because of the $\delta_{e}$-induced coupling.

In undoped QDs it is usual to observe an increase of the Overhauser shift, growing approximately like the electron Zeeman splitting $\left|g_{e} \mu_{\mathrm{B}} B_{z}\right|$, up to fields above $4 \mathrm{~T}$ where it can reach more than $100 \mu \mathrm{eV}$. This growth is interrupted when the depolarization mechanisms get more efficient than the maximum DNP rate obtained for a zero electron-spin splitting (namely when the Overhauser shift gets exactly compensated by the Zeeman effect). This results in an abrupt collapse of the nuclear field which then gives rise to spectral jumps of the $\sigma^{+}$or $\sigma^{-}$PL lines making a clear fingerprint of the DNP vanishing in the magneto-PL images. In Figs. 2(b) and 3(a) these spectral jumps are hardly visible. However, for QD1, by changing slightly the excitation conditions we could create a DNP regime working up to $\approx 2 \mathrm{~T}$ as discussed in the following. For QD2 the splitting $\delta_{e}$ was definitely too large to enable a large Overhauser field to develop, at least in the ranges of temperature, excitation energy, and power we have explored.

\section{DYNAMIC NUCLEAR POLARIZATION IN MN-DOPED QDS}

Figure 4(a) shows the fingerprints of a large Overhauser field which builds up in QD1 when the excitation energy is set to the second resonance $E_{2}$ of Fig. 2(a). The white arrows indicate noticeable jumps of the $\sigma^{+}\left(\sigma^{-}\right)$PL lines towards higher (lower) energies for an increasing magnetic field, or towards lower (higher) energies in a decreasing magnetic field. Let us recall that due to the relative long integration times of each spectrum (10 s) the magnetic field was varied step by step with $50 \mathrm{mT}$ increments, during which the $\sigma^{+}$and $\sigma^{-}$spectra were successively measured. It is therefore normal to observe the jumps at the same magnetic field for a given field sweeping. Conversely, the jumps take place at different magnetic fields, respectively at $1.9 \mathrm{~T}(1.5 \mathrm{~T})$ for an increasing (decreasing) field, revealing the non-Markovian character of the DNP process like observed in undoped QDs [20]. The reason for the drastic change in DNP regime for the two investigated resonances is not clear. Still, we suspect it might be related to the high sensitivity of the DNP mechanism to the effective broadening of the electron spin levels [20], which would be increased when exciting at higher energy.

For Mn-doped QDs, the Overhauser shift is difficult to extract precisely, because of the numerous spectral lines which experience several crossings or anticrossings as a function of the field. Our method to solve this issue consists in determining by a local Gaussian fit the energy of only the $|+1\rangle(|-1\rangle)$ line in negative (positive) fields. In case of ambiguity because of an anticrossing (e.g., near zero field), we retain only the most intense line in order to keep a single line for each spectrum. The energy difference between the same $| \pm 1\rangle$ lines measured in co- and cross-polarized configurations provides, after subtracting a constant exchange energy, the $X^{+}$Zeeman splitting together with the Overhauser shift, but including also noticeable spectral deviations due to the $| \pm 1\rangle$ anticrossings near zero field or $\pm 1.8 \mathrm{~T}$. A reference measurement is thus required to extract properly the sole Overhauser shift. For that purpose, we used the spectra shown in Fig. 1(a) which are
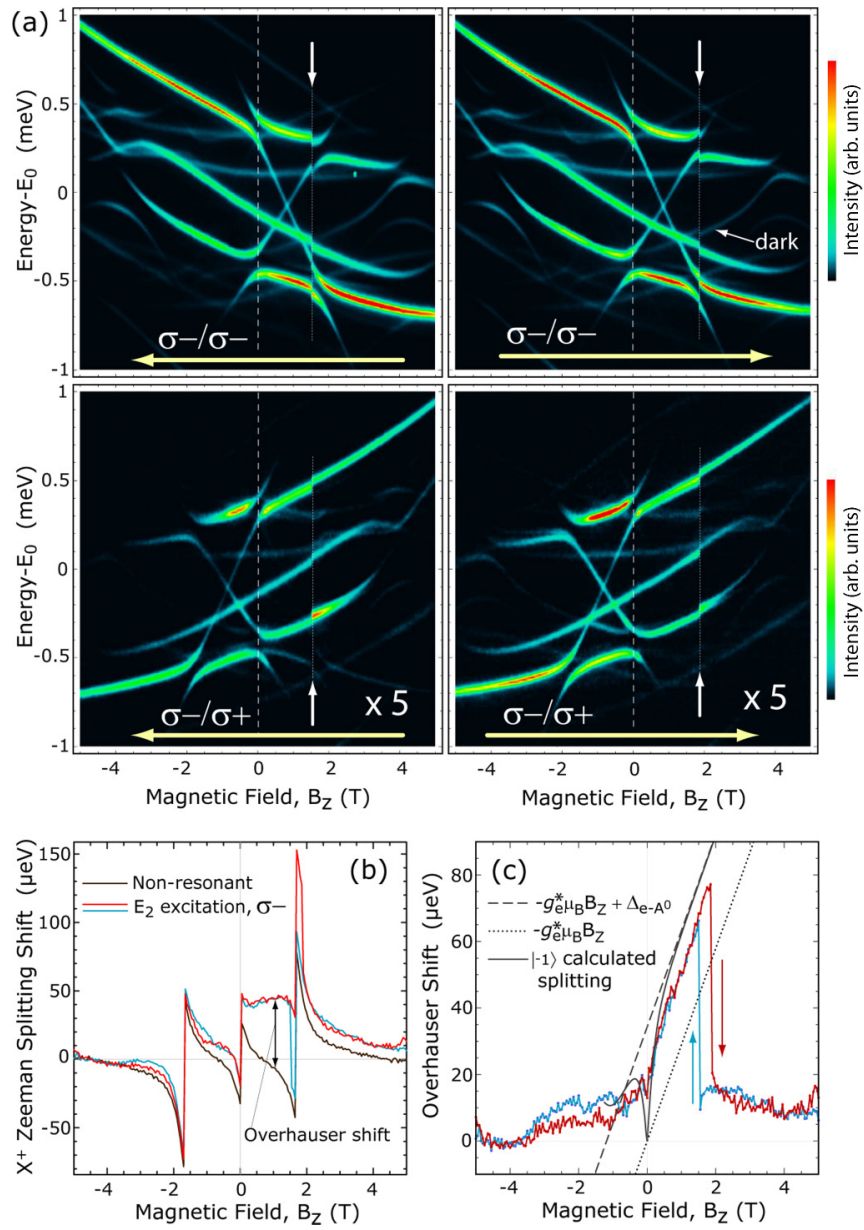

FIG. 4. (a) QD1 PL spectra under quasiresonant $\sigma$ - excitation [resonance $E_{2}$ in Fig. 2(a)] as a function of a decreasing (left) or increasing (right) magnetic field $B_{z}$ and measured in $\sigma-$ (top) or $\sigma+$ (bottom) polarization. (b) Shifts of the linear Zeeman splitting deduced from the dominant $\sigma+$ and $\sigma-$ lines of each spectrum under nonresonant excitation or quasiresonant $\sigma-$ excitation. (c) Overhauser shift deduced from (b) for both field sweep directions.

assumed to be DNP free, since they were performed under nonresonant and nonpolarized excitation.

As an illustration of this procedure, Fig. 4(b) shows the raw splittings of QD1 $\sigma^{+}$and $\sigma^{-}$lines for different measurements and after subtracting the linear slope due to the $X^{+}$Zeeman effect $(\approx 170 \mu \mathrm{eV} / \mathrm{T})$. The $| \pm 1\rangle$ anticrossings give rise to the same specific profile in all measurements which enables us to extract the superimposed Overhauser shift created under circularly polarized excitation as reported in Fig. 4(c). Note that this is the Overhauser shift of the $X^{+}$transition which, besides the dominant shift of the electron Zeeman splitting, can also include a contribution from the hyperfine interaction with the QD-confined hole [30-32]. To assess its relative size, we can compare the energy jumps of the $X^{+}$ bright transitions $(|\uparrow \Downarrow \uparrow\rangle \rightarrow|\Uparrow\rangle)$ with the "dark" transitions $(|\uparrow \Downarrow \uparrow\rangle \rightarrow|\Downarrow\rangle)$. The latter are partially visible thanks to the $A^{0}$-induced coupling of the hole spins [5,6,29]. For QD1 we found that the energy jump of the dark transition marked in Fig. 4(a) is reduced by about $10 \%$ with respect to the jumps of the bright transitions, from which we deduced $a \approx 5 \%$ positive 
contribution of the hole hyperfine interaction to the measured Overhauser shift.

At first glance, the build up of the Overhauser shift in Fig. 4(c) turns out similar to that observed in undoped QDs, with a roughly linear increase in positive fields up to a few Teslas. However, the Overhauser shift lies significantly above the electron Zeeman splitting $-g_{e}^{\star} \mu_{\mathrm{B}} B_{z}$ [dotted line in Fig. 4(c)], where $g_{e}^{\star}=1.05 g_{e}$ is used to take into account the $5 \%$ hole contribution. In undoped QDs, the distance to the Zeeman splitting is usually less than $\simeq 10 \mu \mathrm{eV}$, in order to satisfy the self-consistent condition enabling a high DNP rate over the build up range [20]. Obviously, the relevant electron spin splitting must include the electron- $A^{0}$ exchange which, for the $|-1\rangle$ levels, corresponds to a shift by $B_{\Delta}=\Delta_{e-A^{0}} / g_{e} \mu_{\mathrm{B}} \approx$ $-1 \mathrm{~T}$ of the Zeeman splitting. The agreement in Fig. 4(c) is indeed better with the dashed line $-g_{e}^{\star} \mu_{\mathrm{B}}\left(B_{z}+B_{\Delta}\right)$, or with the theoretical electron spin splitting (solid line) deduced from the $\mid-1$, $\uparrow$ or $\downarrow\rangle$ levels of Fig. 1(b) which also includes the $\delta$ coupling near zero field.

The Overhauser shift remains however below the electron spin splitting by $\simeq 10 \mu \mathrm{eV}$, while in undoped QDs it usually exceeds the Zeeman splitting by about the same amount. We believe this is due to the electron spin depolarization by the $\delta_{e} / 2$ effective coupling which slightly changes the stability point of the DNP. Indeed, the Overhauser shift cannot grow above the total spin splitting (including the Zeeman and exchange terms) because it would require to go through the $\delta_{e}$ anticrossing, where the electron spin projection $S_{e, z}$ vanishes. In other words, when approaching the anticrossing splitting $\delta_{e}$, the DNP rate is drastically reduced, in such a way that the maximum Overhauser shift remains slightly below the electron spin splitting by a few $\delta_{e}$.

Conversely, the $\delta_{e}$-induced depolarization observed in Fig. 2 is significantly changed due to the Overhauser shift. For example, the cross-polarized spectra in Fig. 4(a) no longer exhibit any enhancement around $+1.15 \mathrm{~T}$. The $\mid+1$, $\uparrow\rangle$ and $|+1, \downarrow\rangle$ levels are indeed significantly split by the additional nuclear field in this region and the corresponding $\sigma^{-}$and $\sigma^{+}$ $X^{+}$lines keep a high $\approx 9: 1$ intensity ratio, whereas they have basically the same intensity in Fig. 2(d). More generally, the relative changes in intensity of the lines in Fig. 4(a) seem well correlated to the changes of the measured Overhauser shift which determine the precise splitting of the electron spin states and therefore their actual mixing.

\section{REPLICA OF DNP SEQUENCE DUE TO $A^{0}$-ELECTRON EXCHANGE}

So far we discussed the Overhauser field developing due to electron-nucleus flip-flops taking place between the $\mid-1$, $\uparrow$ or $\downarrow\rangle$ levels. There is an obvious question whether the DNP could take place with the $\mid+1$, $\uparrow$ or $\downarrow\rangle$ levels under the same $\sigma^{-}$polarized excitation. The main difference between the two configurations is that the $\mid+1$, $\uparrow$ or $\downarrow\rangle$ levels become significantly less populated than the $\mid-1$, $\uparrow$ or $\downarrow\rangle$ ones when a positive field increases because of $A^{0}$ thermalization [see Fig. 1(d)]. The DNP is thus expected to be less efficient for $A^{0}$ in $|+1\rangle$ state. In most of the investigated QDs there is indeed no evidence of such contribution. By analyzing the small Overhauser shift of QD1 under excitation at $E_{1}$ energy,
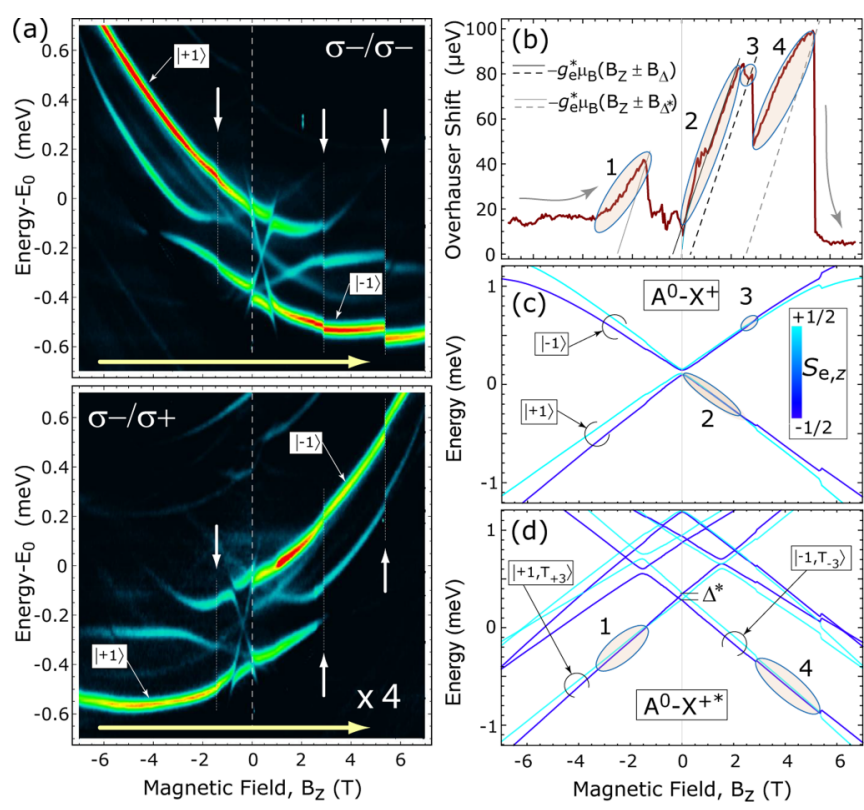

FIG. 5. (a) QD3 PL spectra under quasiresonant $\left(E_{0}+72 \mathrm{meV}\right)$ $\sigma-$ excitation as a function of an increasing magnetic field $B_{z}$ and measured in $\sigma-$ (top) or $\sigma+$ (bottom) polarization $\left(E_{0}=1.304 \mathrm{eV}\right)$. (b) Overhauser shift deduced from (a) and showing four distinct DNP ranges. (c) and (d) Calculated energy levels in the states $A^{0}-X^{+}$(c) and $A^{0}-X^{+^{\star}}$ (d) including the experimental Overhauser shift. Each DNP sequence in (b) can be associated with a pair of closely spaced electron spin levels.

we found possible indications of two distinct increases of DNP field, but with a rather poor signal to noise ratio. Clear DNP replica were however observed for two other quantum dots, QD3 and QD4, as discussed below.

The DNP measurements and analysis of QD3 and QD4 are, respectively, shown in Figs. 5 and 6. Both QDs were quasiresonantly excited at about 2 GaAs LO-phonon energy $(\approx 74 \mathrm{meV})$ above the ground state emission at $\approx 1.3 \mathrm{eV}$ [33]. By sweeping the magnetic field from -6 to $+6 \mathrm{~T}$ under a constant $\sigma^{-}$polarized excitation, we observed several spectral jumps of the QD PL lines, to lower or higher energy depending on the co- or cross-polarized detection configuration, indicating obviously several DNP sequences. By following the same procedure as for QD1, we extracted a quantitative estimate of the Overhauser shift with respect to a DNP-free reference spectrum (not shown). Remarkably, Figs. 5(b) and 6(b) show that both QDs experience successive increases of nuclear polarization, each interrupted by an abrupt, partial, or total fall and exhibiting roughly the same slope.

Observing more than two increases was really not anticipated. Indeed, this cannot be interpreted in the frame of a DNP rate determined by the electron spin splitting of only the $X^{+}-A^{0}$ ground levels. We believe that other levels are required and suggest tentatively in the following that hot trion states $X^{+^{*}}$ could be responsible for the DNP increases starting in high (positive or negative) magnetic fields due to an additional electron-hole exchange energy [34,35]. First, let us focus on the DNP increases starting from a smaller field $\left| \pm B_{\Delta}\right|<2 \mathrm{~T}$ that we ascribe to the $X^{+}-A^{0}$ levels. 

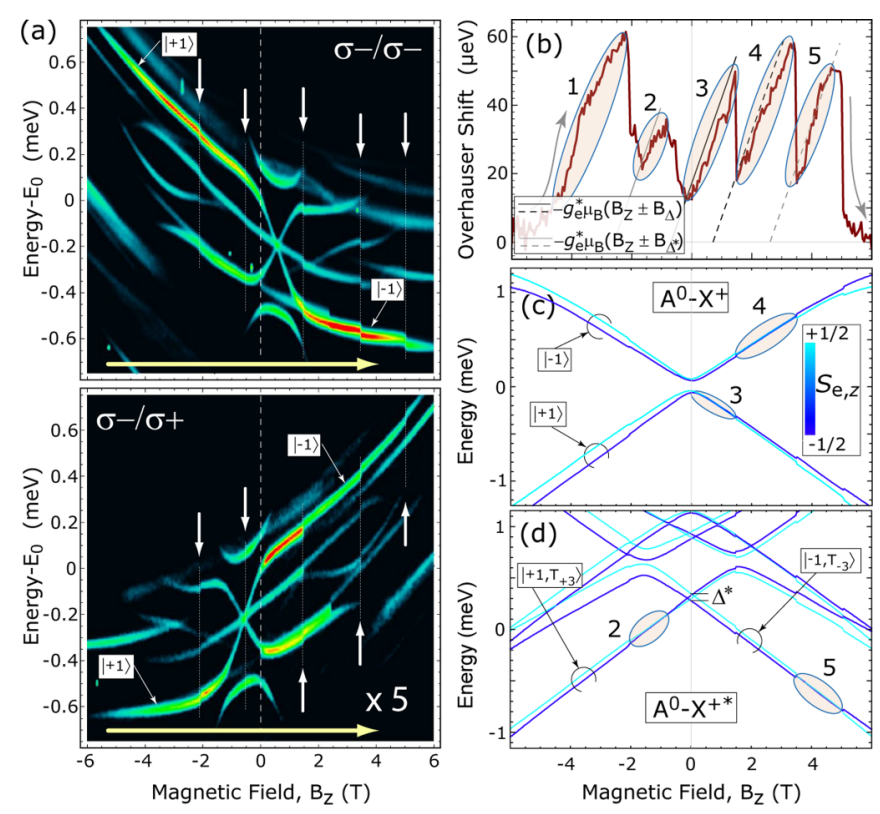

FIG. 6. (a) QD4 PL spectra under quasiresonant $\left(E_{0}+76 \mathrm{meV}\right)$ $\sigma-$ excitation as a function of an increasing magnetic field $B_{z}$ and measured in $\sigma-$ (top) or $\sigma+$ (bottom) polarization $\left(E_{0}=1.307 \mathrm{eV}\right.$ ). (b) Overhauser shift deduced from (a) and showing five successive DNP sequences. (c) and (d) Calculated energy levels in the state $A^{0}-X^{+}$and $A^{0}-X^{+^{\star}}$ including the experimental Overhauser shift. The DNP sequences in (b) can be associated with a pair of closely spaced electron spin levels, but the first increase "1" which would require another trion state.

The theoretical $X^{+}-A^{0}$ levels are plotted in Figs. 5(c) and 6(c) from a spin model using the parameters deduced from the experiments, but including also the measured Overhauser shift. This points out different parts of the $| \pm 1\rangle$ levels (shaded area in the figures) where the actual electron spin splitting is particularly small and therefore enables fast electron-nuclei flip-flops. The relevant $g$ factors and exchange energy of QD3 and QD4, deduced from the anticrossing fields $B_{\delta}$ or the splitting between bright and dark lines, are put together in Table I.

For QD3, the electron- $A^{0}$ exchange is small $(10 \mu \mathrm{eV})$ and thus produces only a small difference of electron spin splitting between the $|+1\rangle$ and $|-1\rangle A^{0}$ states. The DNP associated with the $\mid-1$, $\uparrow$ or $\downarrow\rangle$ levels [labeled "2" in Fig. 5(b)] actually exhibits two regimes: the Overhauser shift first exceeds the electron spin splitting $\approx g_{e}^{\star} \mu_{\mathrm{B}}\left(B_{z}+B_{\Delta}\right)$ in a way similar to undoped QDs, then they cross each other around $1 \mathrm{~T}$ but remain very near in energy up to $2.1 \mathrm{~T}$. This is confirmed by the sudden increase at $1 \mathrm{~T}$ of the $|-1\rangle$ line PL intensity in cross-polarized

TABLE I. Parameters extracted from measurement analysis. Units are $\mathrm{mT}$ for $\left|B_{\delta}\right|$ and $\mu \mathrm{eV}$ for $\Delta_{e-A^{0}}$ and $\delta_{e}$.

\begin{tabular}{lcccccc}
\hline \hline QD & $g_{A^{0}}^{\text {eff }}$ & $\left|B_{\delta}\right|$ & $\Delta_{e-A^{0}}$ & $g_{e}$ & $g_{e}^{\star}$ & $\delta_{e}$ \\
\hline QD1 & 3.6 & 79 & 33 & -0.48 & -0.504 & 9 \\
QD2 & 2.8 & 215 & 72 & -0.57 & - & 47 \\
QD3 & 3.7 & 22 & 10 & -0.52 & -0.556 & 4 \\
QD4 & 3.4 & 50 & 20 & -0.51 & -0.42 & 8 \\
\hline \hline
\end{tabular}

configuration in Fig. 5(a) due to the $\delta_{e}$ induced coupling. Even though this regime is similar to that observed for QD1, the DNP mechanism likely benefits here from the proximity (less than $20 \mu \mathrm{eV}$ ) of the $\mid+1$, $\uparrow$ or $\downarrow\rangle$ spin splitting $\approx g_{e}^{\star} \mu_{\mathrm{B}}\left(B_{z}-B_{\Delta}\right)$. Indeed, above $2.1 \mathrm{~T}$ the Overhauser shift starts a decrease soon interrupted by the DNP sequence " 3 " associated with the $|+1\rangle$ levels. It remains at a rather high level by roughly following the corresponding spin splitting up to $2.9 \mathrm{~T}$, where only it experiences a large reduction by about $30 \mu \mathrm{eV}$.

For QD4, the two DNP increases associated with the two $A^{0}$ states [labeled 3 and 4 in Fig. 6(b)] are even more clearly identified thanks to a larger electron- $A^{0}$ exchange $(20 \mu \mathrm{eV})$ which determines their starting fields $\pm B_{\Delta}$ at $\approx \pm 0.7 \mathrm{~T}$. It is noteworthy that the effective $g$-factor $g_{e}^{\star}$ used to reproduce their slope is smaller (in absolute value) than the actual electron $g$ factor, see Table I. Like for QD1 and QD3, it was determined from the estimate of the hole contribution to the Overhauser shift by comparing the spectral jump of a dark line with respect to a bright line. For QD4 this contribution was surprisingly found to be negative (by about $-17 \%$ ) in contrast to QD1 and QD3. This effect likely results from a very different indium composition of QD4 combined with the different hyperfine coupling constants of the host atomic species, or possibly from a very different strain profile affecting drastically these constants [31]. Further investigations are required to answer this issue and Mn-doped QDs could reveal particularly interesting in that respect by providing almost systematically measurable dark transitions.

\section{TENTATIVE INTERPRETATION OF DNP REPLICA IN HIGH FIELDS}

The increases occurring in higher fields and denoted 1 and 4 in Fig. 5(b) and 1, 2, and 5 in Fig. 6(b) remain truly surprising because they cannot be understood from the level structure in the $A^{0}-X^{+}$configuration. Their slopes, close to $g_{e}^{\star} \mu_{\mathrm{B}}$, still suggest a DNP mechanism based on the compensation by the Overhauser field of an electron spin splitting that would be shifted to higher (positive or negative) fields due to the exchange with a different configuration of the other present spins. Indeed, for both QD3 and QD4 we can trace back a symmetrical origin for two of these DNP increases at the starting field $\pm B_{\Delta^{*}} \approx \pm 2.5 \mathrm{~T}$ which can be ascribed to an exchange energy $\Delta^{\star}=g_{e} \mu_{\mathrm{B}} B_{\Delta^{\star}} \approx 75 \mu \mathrm{eV}$ [see the gray lines in Figs. 5(b) and 6(b)].

To support the interpretation of the high field DNP replica, we considered as a probable candidate an $A^{0}-X^{+^{\star}}$ state, where $X^{+^{*}}$ is a hot $X^{+}$trion having one of its two QDconfined holes occupying a QD excited level. It can be created under nonresonant PL excitation of undoped InGaAs QDs in particular as the intermediate state of a charged biexciton $\left(2 X^{+}\right)$cascade [34-36]. For QD3 we observed a group of spectrally correlated lines, about $3.5 \mathrm{meV}$ below the dominant $A^{0}-X^{+}$features, that we indeed identified as a $2 X^{+} \rightarrow X^{+^{*}}$ transition, thanks to a theoretical simulation of the magneto-PL image, see Fig. 7. It basically consists of two pairs of $| \pm 1\rangle$ lines which can be associated with the $T_{+3}=|\Uparrow \Uparrow\rangle$ or $T_{0}=$ $(|\Uparrow \Downarrow\rangle+|\Downarrow \Uparrow\rangle) / \sqrt{2}$ triplet configurations of a ground state hole $\left(h_{0}\right)$ and an excited state hole $\left(h_{1}\right)$ split by the hole-hole exchange $\Delta_{T}[34,37]$. They each exhibit a specific X-pattern 


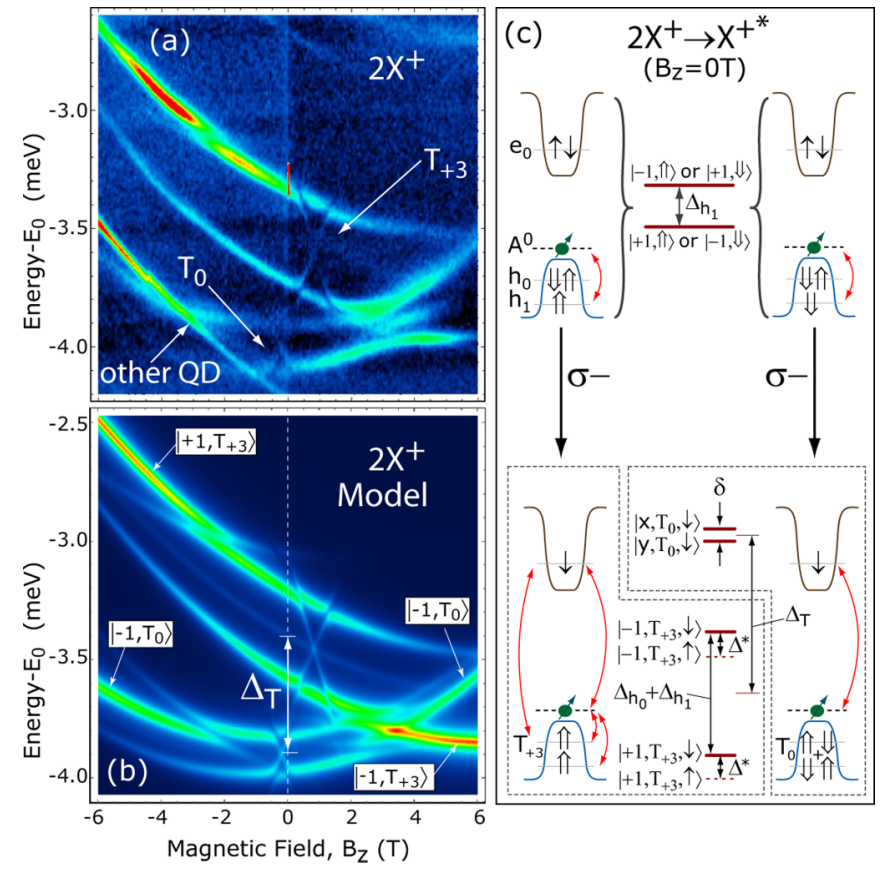

FIG. 7. (a) QD3 PL spectra under quasiresonant $\left(E_{0}+72 \mathrm{meV}\right)$ unpolarized excitation as a function of the magnetic field $B_{z}$ and measured in $\sigma^{-}$circular polarization $\left(E_{0}=1.304 \mathrm{eV}\right)$. (b) Simulated magneto-PL spectra of a $2 X^{+} \rightarrow X^{+^{\star}}$ transition, from a standard spin model. The main fitting parameters are the triplet $T_{0}-T_{ \pm 3}$ splitting $\left(\Delta_{T}=0.5 \mathrm{meV}\right)$, the $A^{0}$ exchange energies with the ground (excited) hole state $\Delta_{h_{0(1)}}=0.4(0.15) \mathrm{meV}$, and the hole $g$-factors $g_{h_{0(1)}}=2.25(-1.05)$. (c) Schematics of the spin levels and exchange interactions (red arrows) involved in a $2 X^{+} \rightarrow X^{+^{\star}} \sigma^{-}$-polarized transition at zero field.

which differs in field position and size. In $T_{+3}$ configuration, the excited $h_{1}$ hole has the same spin in the initial and final state so that its exchange $\Delta_{h_{1}}$ with $A^{0}$ essentially produces a shift in magnetic field by $\Delta_{h_{1}} / g_{A^{0}} \mu_{\mathrm{B}}$ of an X-pattern similar to the $X^{+}$one. In $T_{0}$ configuration, both holes have zero spin projection along $z$ so that their exchange with $A^{0}$ vanishes. The corresponding X-pattern is essentially determined by the $\Delta_{h_{1}}$ exchange in the initial state, see Fig. 7(c). Note that under a fixed polarization detection $\left(\sigma^{-}\right.$here) there is a correlation between the $h_{1} \Uparrow$ or $\Downarrow$ spin and the $T_{+3}$ or $T_{0}$ triplet state [36], which explains the position in positive or negative field of the corresponding X-pattern. Finally, the electron-hole exchange which also contributes to the triplet splitting $\Delta_{T}$, determines with the electron- $A^{0}$ exchange, the electron spin splitting $\Delta^{\star}$ between the bright $\left(\left| \pm 1, T_{+3}, \downarrow\right\rangle\right)$ and dark $\left(\left| \pm 1, T_{+3}, \uparrow\right\rangle\right)$ configurations of $X^{+^{\star}}$ as represented in Fig. 7(c).

Theoretically, this $\Delta^{\star}$ exchange splitting could control the electron-nucleus flip-flop rate in the $X^{+^{*}}$ state and thus explain the additional increases starting from the fields $\pm \Delta^{\star} / g_{e} \mu_{\mathrm{B}}$. However, in contrast to Ref. [34], we could not identify any spectral lines associated with the $X^{+^{*}}$ dark states, and therefore the value of $\Delta^{\star}$ could not be determined experimentally. Therefore, to complete our tentative interpretation we simply adjusted $\Delta^{\star}$ in order to keep the actual electron spin splitting (including the measured Overhauser shift) as small as possible over the field ranges where the increases develop. The corresponding calculated levels are shown in Figs. 5(d) and 6(d) for QD3 and QD4, with shaded areas emphasizing the $\mid \pm 1, T_{ \pm 3}$, $\uparrow$ or $\downarrow\rangle$ levels where the spin splitting is less than $\approx 25 \mu \mathrm{eV}$.

The value of $\Delta^{\star} \approx 75 \mu \mathrm{eV}$ determined in this way turns to be about one order of magnitude smaller than the value reported in Ref. [34] for InAs/GaAs QDs. If such discrepancy is confirmed in future investigations, this might obviously question our specific interpretation based on an $X^{+^{*}}$ state, but the principle should remain valid for another excited state to be identified. This is besides the case of the first DNP increase of QD4 [labeled 1 in Fig. 6(b)] which starts at $\approx-5 \mathrm{~T}$ and therefore cannot be explained by any of the spin splittings calculated for $X^{+}$or $X^{+^{*}}$. Since so far no such effects have been observed with undoped QDs, there is still a strong suspicion that the magnetic impurity is solely responsible for all of the observed DNP increases. This could be due to variations of the electron- $A^{0}$ exchange energy either for an excited electron occupying a different QD orbital, or for an excited $A^{0}$ spin configuration (notably in a $J=2,3$, or 4 spin state).

\section{SUMMARY}

In conclusion, our investigations of the optical orientation of $X^{+}$trions in Mn-doped InGaAs QDs and the subsequent dynamic nuclear polarization have revealed the key role played by the exchange interaction $\Delta_{e}$ between the QD-confined electron and the $\mathrm{Mn}$-induced neutral acceptor state $A^{0}$. On the one hand, the anisotropic part of this exchange gives rise to an effective direct coupling of the electron spin states resulting in the anticrossing $\delta_{e}$ of the corresponding levels and the vanishing of the $X^{+}$spin orientation at the specific magnetic fields $\pm \Delta_{e} / g_{e} \mu_{\mathrm{B}}$. This limits the maximum of the Overhauser field due to the reduction of the electron nuclei flip-flop rate when the Overhauser shift approaches the electron Zeeman splitting. On the other hand, the longitudinal (Ising-like) part of the exchange acts as an effective magnetic field along $z$ giving rise to two successive DNP increases. This analysis is supported by a precise determination of the electron $g$ factor and the contribution of the hole spin to the Overhauser shift, evidencing an energy-driven DNP mechanism similar to that observed for undoped QDs. More surprising is the observation for certain QDs of additional increases in higher positive or negative magnetic fields. We propose an interpretation based on a DNP mechanism taking place in an $X^{+^{\star}}$ state and shifted to a higher fields due to the exchange interaction between the electron and two holes in a triplet $T_{ \pm 3}$ configuration. Still, alternative explanations involving the $A^{0}$ excited states remain plausible and should be more specifically investigated in future works.

\section{ACKNOWLEDGMENTS}

This work was partially supported by "Triangle de la Physique" (project COMAQ), the French RENATECH network, and a public grant overseen by the French National Research Agency (ANR) as part of the "Investissements d'Avenir" program (Labex NanoSaclay, reference: ANR-10LABX-0035). 
[1] L. Besombes, Y. Léger, L. Maingault, D. Ferrand, H. Mariette, and J. Cibert, Phys. Rev. Lett. 93, 207403 (2004).

[2] Y. Léger, L. Besombes, L. Maingault, D. Ferrand, and H. Mariette, Phys. Rev. Lett. 95, 047403 (2005).

[3] Y. Léger, L. Besombes, J. Fernandez-Rossier, L. Maingault, and H. Mariette, Phys. Rev. Lett. 97, 107401 (2006).

[4] Y. Léger, L. Besombes, L. Maingault, and H. Mariette, Phys. Rev. B 76, 045331 (2007).

[5] A. Kudelski, A. Lemaître, A. Miard, P. Voisin, T. C. M. Graham, R. J. Warburton, and O. Krebs, Phys. Rev. Lett. 99, 247209 (2007).

[6] O. Krebs, E. Benjamin, and A. Lemaître, Phys. Rev. B 80, 165315 (2009).

[7] A. H. Trojnar, M. Korkusiński, E. S. Kadantsev, P. Hawrylak, M. Goryca, T. Kazimierczuk, P. Kossacki, P. Wojnar, and M. Potemski, Phys. Rev. Lett. 107, 207403 (2011).

[8] A. H. Trojnar, M. Korkusinski, U. C. Mendes, M. Goryca, M. Koperski, T. Smolenski, P. Kossacki, P. Wojnar, and P. Hawrylak, Phys. Rev. B 87, 205311 (2013).

[9] U. C. Mendes, M. Korkusinski, A. H. Trojnar, and P. Hawrylak, Phys. Rev. B 88, 115306 (2013).

[10] C. Le Gall, L. Besombes, H. Boukari, R. Kolodka, J. Cibert, and H. Mariette, Phys. Rev. Lett. 102, 127402 (2009).

[11] C. Le Gall, R. S. Kolodka, C. L. Cao, H. Boukari, H. Mariette, J. Fernández-Rossier, and L. Besombes, Phys. Rev. B 81, 245315 (2010).

[12] C. Le Gall, A. Brunetti, H. Boukari, and L. Besombes, Phys. Rev. Lett. 107, 057401 (2011).

[13] E. Baudin, E. Benjamin, A. Lemaître, and O. Krebs, Phys. Rev. Lett. 107, 197402 (2011).

[14] M. Goryca, T. Kazimierczuk, M. Nawrocki, A. Golnik, J. A. Gaj, P. Kossacki, P. Wojnar, and G. Karczewski, Phys. Rev. Lett. 103, 087401 (2009).

[15] D. E. Reiter, T. Kuhn, and V. M. Axt, Phys. Rev. Lett. 102, 177403 (2009).

[16] D. E. Reiter, T. Kuhn, and V. M. Axt, Phys. Rev. B 83, 155322 (2011).

[17] L. Besombes, C. L. Cao, S. Jamet, H. Boukari, and J. FernándezRossier, Phys. Rev. B 86, 165306 (2012).

[18] O. Krebs and A. Lemaître, Phys. Rev. Lett. 111, 187401 (2013).

[19] J. Kobak, T. Smoleński, M. Goryca, M. Papaj, K. Gietka, A. Bogucki, M. Koperski, J.-G. Rousset, J. Suffczyński, E. Janik, M. Nawrocki, A. Golnik, P. Kossacki, and W. Pacuski, Nat. Commun. 5, 3191 (2014).
[20] B. Urbaszek, X. Marie, T. Amand, O. Krebs, P. Voisin, P. Maletinsky, A. Högele, and A. Imamoglu, Rev. Mod. Phys. 85, 79 (2013).

[21] B. Eble, O. Krebs, A. Lemaître, K. Kowalik, A. Kudelski, P. Voisin, B. Urbaszek, X. Marie, and T. Amand, Phys. Rev. B 74, 081306(R) (2005).

[22] P.-F. Braun, B. Urbaszek, T. Amand, X. Marie, O. Krebs, B. Eble, A. Lemaître, and P. Voisin, Phys. Rev. B 74, 245306 (2006).

[23] A. I. Tartakovskii, T. Wright, A. Russell, V. I. Fal'ko, A. B. Van'kov, J. Skiba-Szymanska, I. Drouzas, R. S. Kolodka, M. S. Skolnick, P. W. Fry, A. Tahraoui, H.-Y. Liu, and M. Hopkinson, Phys. Rev. Lett. 98, 026806 (2007).

[24] P. Maletinsky, C. W. Lai, A. Badolato, and A. Imamoglu, Phys. Rev. B 75, 035409 (2007).

[25] E. A. Chekhovich, M. N. Makhonin, K. V. Kavokin, A. B. Krysa, M. S. Skolnick, and A. I. Tartakovskii, Phys. Rev. Lett. 104, 066804 (2010).

[26] W. Yang and L. J. Sham, Phys. Rev. B 88, 235304 (2013).

[27] J. Schneider, U. Kaufmann, W. Wilkening, M. Baeumler, and F. Köhl, Phys. Rev. Lett. 59, 240 (1987).

[28] F. Marczinowski, J. Wiebe, J.-M. Tang, M. E. Flatté, F. Meier, M. Morgenstern, and R. Wiesendanger, Phys. Rev. Lett. 99, 157202 (2007).

[29] M. Goryca, P. Plochocka, T. Kazimierczuk, P. Wojnar, G. Karczewski, J. A. Gaj, M. Potemski, and P. Kossacki, Phys. Rev. B 82, 165323 (2010).

[30] E. A. Chekhovich, A. B. Krysa, M. S. Skolnick, and A. I. Tartakovskii, Phys. Rev. Lett. 106, 027402 (2010).

[31] E. A. Chekhovich, A. B. Krysa, M. Hopkinson, P. Senellart, A. Lemaître, M. S. Skolnick, and A. I. Tartakovskii, Nat. Phys. 9 74 (2013).

[32] P. Fallahi, S. T. Yilmaz, and A. Imamoglu, Phys. Rev. Lett. 105 , 257402 (2010).

[33] A lower excitation at about 1 LO phonon, like for QD1, gave the same results for QD3, but was unable to create efficiently the $X^{+}$state in QD4.

[34] T. Warming, E. Siebert, A. Schliwa, E. Stock, R. Zimmermann, and D. Bimberg, Phys. Rev. B 79, 125316 (2009).

[35] E. Siebert, T. Warming, A. Schliwa, E. Stock, M. Winkelnkemper, S. Rodt, and D. Bimberg, Phys. Rev. B 79 205321 (2009).

[36] E. Poem, Y. Kodriano, C. Tradonsky, B. D. Gerardot, P. M Petroff, and D. Gershoni, Phys. Rev. B 81, 085306 (2010).

[37] M. Ediger, G. Bester, B. D. Gerardot, A. Badolato, P. M. Petroff, K. Karrai, A. Zunger, and R. J. Warburton, Phys. Rev. Lett. 98, 036808 (2007). 